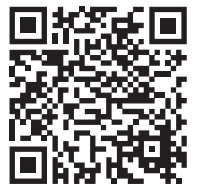

\footnotetext{
Palabras clave: Simulación con pacientes, artículo de revista, entrenamiento en simulación, publicación.

Keywords: Patient simulation, journal article, simulation training, publishing.
}

* Profesor Asociado, División de

Anestesiología.

‡ Interno de Medicina.

$\S$ Instructor adjunto.

Departamento de

Traumatología.

División de Cirugía.

Escuela de Medicina,

Facultad de Medicina,

Pontificia

Universidad

Católica de Chile.

Recibido: 18/02/2021

Aceptado: 25/03/2021

doi: $10.35366 / 99866$

\section{Cómo diseñar y escribir un protocolo de investigación basado en simulación}

\author{
How to design and write a simulation-based research protocol
}

\author{
Claudio Nazar J,* Nicole Bloch G, ${ }^{\ddagger}$ Lucas Oberpaur K, ${ }^{\ddagger}$ Daniela Regonesi $\mathrm{P},{ }^{\ddagger}$ \\ Francisca Lubi R, ${ }^{\ddagger}$ Pablo Besa $\mathrm{V}, \S$ Marcia Corvetto A*
}

\section{RESUMEN}

La investigación basada en simulación ha crecido de manera exponencial en los últimos años; sin embargo, creemos que la calidad de los artículos debe mejorar. Para ello, es fundamental planificar una investigación de manera estructurada desde sus inicios. Primero, se debe establecer una pregunta de investigación adecuada, luego justificar el motivo del estudio, definir los desenlaces relevantes y trazar el diseño de la investigación. Una vez desarrollada la investigación se debe redactar el artículo considerando la estructura, que incluye título, resumen, palabras clave, introducción, material y métodos, resultados, discusión, conclusiones, agradecimientos, referencias y datos suplementarios o anexos. También se debe considerar el lenguaje a utilizar, siendo correcto, breve y preciso. El propósito de este trabajo es servir como guía práctica para facilitar el diseño y la escritura de un protocolo de investigación basada en simulación.

\section{ABSTRACT}

Simulation based research has seen exponential growth in recent years, but the quality of such articles needs improvement. To achieve that goal, structured planning of simulation based research protocols is key. First, one must establish an adequate clinical question and justify the reasons for researching the subject. Afterwards, significant outcomes must be selected in order to start designing the protocol itself. Once the research has been conducted, an article must be written that considers general structure determined by scientific literature standards. It includes title, abstract, key words, introduction, materials and methods, results, discussion, conclusions, acknowledgements, references and supplementary data. The language to be used is also to be considered, since it has to be correct, brief and precise. The purpose of this paper is to be used as a guideline to ease the process of designing and writing a simulation based research protocol.

\section{INTRODUCCIÓN}

E n los últimos años la simulación clínica ha crecido de modo exponencial. Esto nos lleva a plantearnos la manera más adecuada de plasmar los resultados obtenidos de la investigación basada en simulación.

Para poder realizar trabajos de investigación de calidad resulta fundamental plantear preguntas de investigación apropiadas, que luego se intentan responder a través de diseños de investigación pertinentes, para por último plasmar en el papel los diferentes resultados obtenidos a través de una que se dispone para comunicar nuevos conocimientos en la comunidad científica para que otros puedan basarse en ellos y dar los siguientes publicación. En la actualidad, esta es la manera pasos. De este modo se crece académicamente. Además, si una persona se vuelve conocida por sus trabajos, puede ser citado por otros y dar paso a nuevos estudios. También puede optar a fuentes de financiamiento para sus proyectos, elevar la calidad de su investigación y otorgar prestigio a la institución a la cual pertenece. Por ende, publicar es una forma efectiva de comunicar conocimiento y avanzar en la carrera académica. ${ }^{1}$

En términos generales, la investigación basada en simulación (IBS) se puede dividir en dos tipos de estudios. En primer lugar se encuentran los estudios que evalúan la efectividad de la simulación como metodología de enseñanza-aprendizaje, es decir, la simulación en el sujeto a estudiar. Un ejemplo es un estudio que compara las habilidades de reanimación cardiopulmonar (RCP)
Citar como: Nazar JC, Bloch GN, Oberpaur KL, Regonesi PD, Lubi RF, Besa VP, et al. Cómo diseñar y escribir un protocolo de investigación basado en simulación. Rev Latinoam Simul Clin. 2021; 3 (1): 28-34. https://dx.doi.org/10.35366/99866 
adquiridas por alumnos de pregrado de Medicina luego de una instrucción mediante una plataforma computacional versus una instrucción con simulación de alta fidelidad. En segundo lugar, están aquellos estudios que usan la simulación como metodología de investigación, esto es, como un medio ambiente en el cual se desarrolla una investigación. ${ }^{2,3}$ Por ejemplo, un estudio que investiga si la adherencia a los protocolos de reanimación cardiopulmonar es mejor al usar ayudas cognitivas versus no usarlas, y utiliza el ambiente simulado para su desarrollo, ya que en la vida real sería antiético hacerlo.

La IBS ha crecido rápidamente durante los último años; sin embargo, la calidad de los reportes es variable y debe mejorar. ${ }^{4}$ Estas mejoras se pueden dar con un aprendizaje relativamente rápido, ya que muchas de las características de las IBS son similares a la investigación clínica o del área de la educación. ${ }^{2}$ En este contexto, el objetivo de este manuscrito es otorgar una guía práctica para diseñar y, posteriormente, escribir un protocolo de investigación en simulación clínica, para finalmente lograr una publicación en una revista científica afín al tema.

\section{RECOMENDACIONES PARA DISEÑAR UN PROTOCOLO DE INVESTIGACIÓN BASADO EN SIMULACIÓN CLÍNICA}

Al desarrollar un protocolo de IBS, se recomienda tener en cuenta una serie de pasos a seguir:

1. Pregunta de investigación: en primer lugar, resulta fundamental tomarse el tiempo de hacer una buena pregunta de investigación. Antes de definir cuál será la pregunta de investigación, es importante tener claro que debe tener una serie de atributos. La pregunta debe ser factible de realizar en cuanto a su alcance, experticia, recursos y reclutamiento necesarios. También debe ser atractiva y novedosa, de manera que despierte el interés del investigador y su equipo, y de la comunidad científica. Es importante que la pregunta sea nueva, en el sentido de que no esté respondida ya en la literatura actual, puesto que si es una pregunta que ya tiene respuesta previa, probablemente será un estudio difícil de publicar en una revista indexada. Es necesario que sea ética, se debe tener en cuenta que una investigación que presente riesgos inaceptables o violación a la privacidad no puede realizarse. Por último, uno de los puntos más importantes es que la pregunta sea relevante, al considerar que aporte nuevos conocimientos científicos, a la práctica clínica, a la salud pública o sirva como base para futuras investigaciones. ${ }^{5}$ Al momento de formular una pregunta de investigación se utiliza la nemotecnia PICO, que describe los diferentes componentes de la pregunta. En primer lugar, la letra $\mathrm{P}$ se refiere a cuál es la población o el problema que se desea estudiar. Luego, la letra I especifica la intervención o exposición a evaluar. Por ejemplo, si se desea evaluar un nuevo protocolo de entrenamiento en simulación. En tercer lugar, la C corresponde a cuál será la comparación, ya sea con el estándar actual, otra intervención novedosa, o bien ausencia de intervención/placebo. Por ejemplo, comparar dos tipos diferentes de feedback (estructurado versus no estructurado) en un entrenamiento simulado. Finalmente, la letra $\mathrm{O}$ se refiere a los outcomes o desenlaces que se medirán, entre los cuales se consideran la eficacia, seguridad, diferentes parámetros clínicos, etcétera. Existen algunos autores que además han agregado una letra $\mathrm{T}$ al final, quedando la nemotecnia PICOT, que se refiere al tiempo en que se evaluará el estudio, ya sea en un punto fijo en el tiempo o seguimiento continuo por un plazo determinado. ${ }^{6}$ Esta forma de establecer una pregunta de investigación es universal a la investigación en salud de todo tipo.

2. Justificación: es importante plasmar en el artículo la razón o el porqué se realizó la investigación. Esto implica una revisión de la literatura publicada al respecto, incluyendo la literatura gris y la búsqueda de ensayos en desarrollo. Mucha de la literatura previa de simulación se encuentra en revistas no indexadas o en revistas indexadas, pero de otras áreas de la educación médica y salud. Considera también una crítica de esta literatura encontrada, destacando eventuales discrepancias entre estudios y la metodología utilizada en éstos. El propósito final es justificar y fundamentar la investigación propuesta desde un punto de vista científico, económico y ético. ${ }^{6}$

3. Desenlaces o outcomes: son parte de la pregunta de investigación (letra O de la nemotecnia explicada anteriormente) y uno de los puntos claves a decidir. Se deben elegir aquellos desenlaces que sean relevantes, medibles y que tengan una asociación plausible 
con la intervención. Habitualmente, en la IBS caen en una de las siguientes tres categorías: desenlaces medidos a través del simulador, pautas observacionales y desenlaces clínicos. Los resultados medidos a través del simulador pueden llegar a ser muy precisos, como por ejemplo el tiempo que transcurre hasta que se realizan adecuadamente una toma de pulso o compresiones torácicas durante una RCP. Sin embargo, se debe tomar en consideración cuál es el real significado clínico del desenlace a medir. Las pautas observacionales pueden ser muy útiles para evaluar habilidades técnicas, conductas y desempeño clínico, pero se debe asegurar la validez interna y externa de estas listas para mejorar la calidad del estudio. Por ello, es recomendable diseñar la o las herramientas para cada estudio y previamente realizar una prueba piloto para probar la aplicabilidad de ésta(s). Los desenlaces clínicos, a pesar de tener el mayor peso, son difíciles de medir mediante simulación, ya que el grado de correlación entre el desempeño en un simulador versus con el paciente real no es del todo conocido. ${ }^{3}$

4. Diseño: una vez que la pregunta de investigación está lista, se debe elegir el mejor diseño de investigación que permita responderla. La elección del diseño de un estudio es una de las etapas más complejas en el proceso de investigación. Con respecto a los diseños de investigación, distintos textos ofrecen diferentes clasificaciones y explicaciones. ${ }^{7-10}$ En este manuscrito se utilizará el que nos parece más simple y ejemplificador para los estudios en simulación, teniendo en cuenta que estas clasificaciones fueron descritas para estudios clínicos. ${ }^{8}$ A continuación, se describirán los diseños para estudios cuantitativos (Figura 1).

Lo primero que debe decidir el investigador es definir si se mantendrá al margen del desarrollo de los acontecimientos o decidirá intervenir en ellos. Es decir, si el estudio será observacional o experimental, según si el investigador realiza o no una intervención.

Los estudios observacionales corresponden a diseños de investigación cuyo objetivo es «la observación» de acontecimientos sin intervenir en el curso natural de éstos. ${ }^{8}$ Por lo tanto, en este tipo de estudios no existe ninguna intervención por parte del investigador.

Los estudios observacionales se subdividen en dos tipos: descriptivos y analíticos. En un estudio observacional descriptivo lo que se pretende es "describir y registrar" lo observado, como el comportamiento de una o más variables en un grupo de sujetos en un periodo de tiempo. Por ejemplo, un relato de cómo se realiza un programa de entrenamiento simulado en alumnos de pregrado de Medicina de cuarto año es descriptivo y no tiene hipótesis. Los estudios observacionales analíticos son aquellos que permiten "comparar grupos de sujetos" sin que exista un proceso de asignación de los individuos en estudio a una intervención determinada, por ende, el investigador es un mero observador y descriptor de lo que ocurre. Se aplican muy bien a estudios epidemiológicos. Un ejemplo en simulación sería una medición del grado de satisfacción del personal de un hospital público después de un curso de RCP básica.

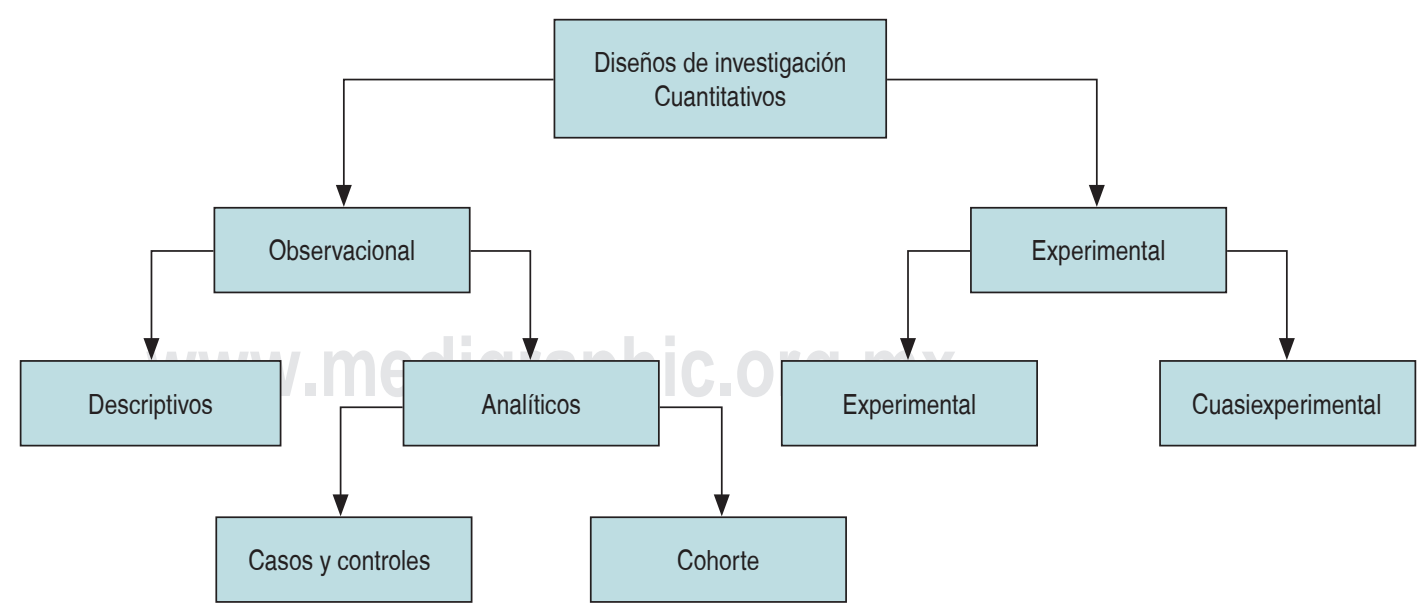

Figura 1: Tipos de diseños para investigación cuantitativa. 
Tabla 1: Estructura general de un artículo científico en simulación.

1. Título

2. Resumen o abstract

3. Palabras clave o keywords

4. Texto principal

4.1. Introducción

4.2. Material y métodos

4.3. Resultados

4.4. Discusión

5. Conclusiones

6. Reconocimientos

7. Datos suplementarios y anexos

En los estudios experimentales el investigador realiza una intervención o "experimento". Su mayor exponente es el ensayo clínico aleatorizado o randomized controlled trial (RCT), en el que se generan grupos iguales en todo y es el azar el que determina la exposición o no a la intervención (experimental real). Un ejemplo en simulación es dividir al azar a alumnos de pregrado de Medicina a dos tipos distintos de feedback durante un taller de paracentesis: feedback del experto versus feedback de pares.

Es importante mencionar a los estudios cuasiexperimentales, ya que son un diseño frecuente en simulación. ${ }^{11}$ Ellos por definición son estudios experimentales, dentro de los cuales algunos subtipos son: pretest-postest de un solo grupo; el de grupo no equivalentes sin pretest; el de grupo control no equivalente; el de grupo control no equivalente pretest y postest; y el de series temporales interrumpidas. Un ejemplo de pretest-postest de un solo grupo es la medición de habilidades técnicas y no técnicas en instalación de catéter venoso central, antes y después de un entrenamiento en simulación en residentes de primer año de Anestesiología.

Una vez elegido el diseño del estudio, hay muchos detalles importantes a considerar para poder evitar variables confundentes que son particulares de la IBS, tanto para estudios que evalúan la efectividad de la simulación como para los que la usan como herramienta de investigación. Una variable confundente es aquélla que no fue controlada o considerada por el investigador y que altera la validez interna del estudio, dado que puede provocar que se analicen los resultados de manera incorrecta. ${ }^{12}$ Para evitarlas se debe planear con cuidado y antelación el escenario de simulación, su implementación y el registro de información. De tal manera, se debe reproducir exacta y correctamente el mismo escenario repetidas veces para los distintos sujetos $y$, en caso de tratarse de un estudio multicéntrico, entre los distintos centros. La forma de registrar la información muchas veces es favorecida por el uso de cámaras de audio y video, de manera que permite el desarrollo más libre de la simulación mientras aumenta la consistencia y precisión de la evaluación.

\section{RECOMENDACIONES PARA ESCRIBIR EL ARTÍCULO}

Un artículo científico de simulación, al igual que uno de investigación clínica, tiene una estructura clásica, la cual se ve representada en la Tabla 1. ${ }^{13}$ Existen algunos artículos que no llevan este formato, como artículos de reflexión, opiniones de experto, conceptos y comentarios, para lo cual uno debe acogerse a las instrucciones para los autores de cada revista en específico.

1. Título: es fundamental para guiar a quien lee y busca el trabajo. Es por esto que debe ser atractivo y tener la menor cantidad de palabras posibles que describan el contenido. ${ }^{14}$ Uno debe preguntarse: ¿es informativo y refleja el tema principal del estudio? Se debe evitar usar tanto un título muy general como uno demasiado específico. El primero confunde respecto al tema que tratará el artículo y el segundo entrega tanta información que puede desalentar al lector. Además, se debe evitar el uso de abreviaciones, nombres registrados y terminología poco ortodoxa. ${ }^{15}$

2. Resumen o abstract: se deben exponer las ideas y los resultados esenciales del artículo con el fin de ofrecer una imagen del contenido y valor del trabajo. Su propósito es despertar el interés del lector por la lectura total del artículo. ${ }^{14}$ Debe ser preciso, conciso y entenderse por sí solo sin necesidad de leer el artículo completo, ya que puede actuar como sustituto del texto si no se dispusiera de él. ${ }^{16}$ Debe escribirse en tercera persona y en pasado. Su longitud es generalmente entregada por cada revista en las instrucciones para los autores. Es recomendable seguir la misma estructura del artículo: describir la metodología, resumir los resultados y presentar las principales conclusiones, por lo que es importante escribirlo una vez terminado el artículo. ${ }^{14}$ Los errores más frecuentes en su 
redacción son hacerlo excesivamente detallado y/o demasiado extenso. ${ }^{15}$

3. Palabras clave o keywords: de manera frecuente están escritas bajo el resumen. Son de gran utilidad para clasificar el artículo en las bases de datos electrónicas y para su búsqueda online. ${ }^{17}$ Se deben elegir con cuidado, dado que emplear cualquier palabra clave podría quitarle visibilidad al artículo y evitar que se encuentre disponible para los lectores cuando busquen información en las distintas bases de datos electrónicas existentes. El número de palabras clave a utilizar suele ser entre tres y 10 . Se recomienda utilizar de preferencia los términos MeSH (Medical Subject Headings) de PubMed como keywords. ${ }^{15}$

4. Texto principal: el texto principal de un artículo de IBS por lo general incluye cuatro partes: introducción, material y métodos, resultados y discusión.

4.1 Introducción: La introducción cumple el rol de responder la pregunta de "ipor qué se ha hecho este trabajo?". ${ }^{15}$ Debe incluir una breve reseña de lo que se sabe actualmente sobre el tema en cuestión, pudiendo incluir otros artículos relacionados, así como también los conocimientos faltantes..$^{18}$ Finalmente, en el último párrafo se debe presentar el objetivo principal del estudio. ${ }^{14,17}$ Es importante evitar una extensión excesiva, siendo recomendable utilizar tres o cuatro párrafos, y debe ser redactada en presente. ${ }^{14,15}$

4.2 Material y métodos: luego de la introducción, sigue la sección de material y métodos. Es una de las partes más importantes del artículo, ya que de acuerdo a ella será juzgada la validez interna y externa de éste. Debe ser escrita con suficiente orden y detalle como para que otros puedan repetir el estudio y el lector sea capaz de juzgar si los resultados son válidos. Considera las distintas partes que comprenden la pregunta de investigación (nemotecnia PICO). La población debe ser descrita en cuanto a sus criterios de selección, datos demográficos y antecedentes clínicos que fuesen relevantes. Los materiales y su preparación requieren una adecuada descripción, así como la estructura del protocolo de intervención y cómo se realizan las distintas mediciones. Por último, hay que reflejar cuáles fueron los cálculos realizados y las herramientas estadísticas utilizadas. Se redacta en pasado y con lenguaje preciso. ${ }^{19}$

4.3 Resultados: los resultados deben ser presentados de forma breve y clara. ${ }^{16}$ Deben cumplir con dos funciones: revelar los resultados de las experiencias descritas en material y métodos, y entregar las pruebas que apoyan los resultados entregados. Las pruebas pueden ser entregadas a través de figuras, tablas o texto. Se recomienda que el primer párrafo sea utilizado para resumir en una frase clara y concisa el hallazgo principal del estudio. ${ }^{15}$ Luego, se recomienda seguir el orden establecido anteriormente en la sección de material y métodos. De esta forma, se puede comenzar con una descripción general de la muestra para posteriormente ceñirse a los resultados de interés. ${ }^{15}$ Es fundamental que todas las tablas y figuras presentadas sean citadas en el artículo, comentando en el texto correspondiente los datos más relevantes de éstas. El objetivo de las tablas y figuras es que se pueda comprender lo más importante de los resultados, evitando la redundancia y sin que sea imprescindible consultarlo nuevamente en el texto. ${ }^{14}$ Esta sección debe ser escrita utilizando los verbos en pasado. ${ }^{16}$

4.4 Discusión: finalmente, la última sección del texto principal es la discusión. En ella se deben comentar los resultados más relevantes del estudio, resaltando los aspectos nuevos e importantes obtenidos, y $\sin$ repetir los datos u otro material presentado con anterioridad. ${ }^{16}$ Todos los resultados presentados previamente deben ser discutidos, cabe señalar que no es apropiado presentar resultados adicionales que no fueron entregados anteriormente. ${ }^{19}$ Es necesario establecer nexos entre los objetivos planteados y los resultados obtenidos, realizando también una correlación de los hallazgos del estudio con la evidencia ya existente. ${ }^{14,15}$

Algunas de las recomendaciones para esta sección son: comenzar dando una respuesta a la pregunta y objetivos planteados en la introducción, siguiendo con las pruebas que fueron expuestas en los resultados que permitan o no corroborarla. ${ }^{15}$ Además, es importante escribir esta sección en presente, dado que los hallazgos del trabajo son ya considerados 
evidencia científica. ${ }^{14}$ Es importante identificar los errores metodológicos (sesgos) y los resultados anómalos, dándoles una explicación lo más coherente posible o mencionando lo que se ha encontrado, aunque no tenga una explicación por el momento. ${ }^{14,15}$ La discusión es la sección más compleja de elaborar y de organizar. Aquella investigación que entregue resultados coherentes y una buena discusión, probablemente asegura su publicación.

5. Conclusiones: no debe ser un resumen del artículo. En ella se realizan las declaraciones finales con base en los argumentos expuestos en la sección de discusión. ${ }^{13}$ Sobre la base de los hallazgos del artículo, en esta sección puede agregarse una declaración sobre los potenciales cambios a la práctica clínica o las posibles oportunidades para futuras investigaciones, pero no deben incluirse especulaciones ni material adicional al discutido en las secciones previas. ${ }^{19}$ Es recomendable que la sección de conclusiones sea corta, de preferencia no mayor a un párrafo. ${ }^{13}$

6. Reconocimientos: Permite la publicación de los nombres de aquellos individuos o instituciones que permitieron la realización de la publicación, pero que no son coautores. También es importante mencionar las fuentes de financiamiento en el caso que sea pertinente. $^{18}$

7. Datos suplementarios y anexos: la utilización de figuras puede ser indispensable para presentar procesos complejos o para describir imágenes que costaría mucho esfuerzo describir con palabras. ${ }^{16}$ Una figura incluye todo tipo de material no tabular (morfología, algoritmos, histogramas, gráficas, fotografías, etcétera). Las figuras deben tener una leyenda con una descripción detallada en la que se explique cada símbolo utilizado. ${ }^{18}$ La fuente debe ser clara y uniforme entre todas las figuras. Éstas deben ser ordenadas y fáciles de comprender. ${ }^{13}$ La tabla tiene la ventaja de mostrar mejor los valores numéricos exactos con sus posibles interrelaciones, mientras que un gráfico expresa mejor la tendencia de los datos o patrones bien definidos. Es por esto que la tabla se utilizará cuando la precisión de los datos es importante y el gráfico cuando los datos presentan una tendencia definida o permiten resaltar una diferencia. ${ }^{14}$ En el caso de usar tablas, éstas deben ser capaces de transmitir su mensaje sin confundir al lector, ${ }^{13}$ deben tener un título claro, preciso y conciso, y los datos se han de organizar de arriba hacia abajo. ${ }^{15}$ Finalmente, al usar figuras y tablas complementarias debe evitarse duplicar la información expuesta en el cuerpo o texto del artículo. ${ }^{18}$

\section{CONCLUSIÓN}

El creciente uso de la IBS evidencia su amplia utilidad en el desarrollo de nuevos conocimientos, destrezas clínicas y un sinnúmero de funciones por descubrir. Sin embargo, su uso requiere de la comprensión tanto de sus beneficios como de los desafíos particulares que ésta presenta. Por lo tanto, al momento de desarrollar un protocolo de IBS se debe considerar una serie de pasos a seguir para evitar un detrimento de la validez interna y externa del estudio, y elegir el mejor diseño para el proyecto en particular.

Asimismo, todo esfuerzo es en vano si no se logran comunicar de manera efectiva y atractiva los nuevos conocimientos generados. Por lo tanto, al momento de escribir el artículo de investigación es imperativo atenerse a las normas de estructura establecidas en la literatura científica, complementada con un lenguaje que sea claro, conciso y fácil de entender, a manera de cautivar al lector y también al editor de la revista a la cual se esté postulando para publicar.

El trabajo de planificar, desarrollar y redactar un protocolo de IBS es un proceso largo y desafiante, pero que realizado correctamente puede aportar nuevos conocimientos que beneficiarán al personal de salud y, más importante, a nuestros pacientes. Es por ello que en este artículo los autores plantean una serie de pasos, a modo de referencia, para facilitar el aprendizaje de este proceso y lograr trabajos de mayor calidad que logren reflejar el esfuerzo y el conocimiento obtenido, entregándolo fidedignamente al lector. Es importante destacar que estos pasos no pretenden ser una regla universal y deben ajustarse caso a caso según el tipo de investigación que se realiza.

\section{REFERENCIAS}

1. Hoogenboom B, Manske R. Invited commentary how to write a scientific article. The International Journal of Sports Physical Therapy. 2012; 7 (5): 512-517.

2. Cheng A, Auerbach M, Hunt EA, et al. Designing and conducting simulation-based research. Pediatrics. 2014; 133 (6): 1091-1101.

3. Cheng A, Kessler D, Mackinnon R, et al. Reporting guidelines for health care simulation research: 
extensions to the CONSORT and STROBE statements. Simul Healthc. 2016; 11 (4): 238-248.

4. Cook DA, Hatala R, Brydges R, et al. Technologyenhanced simulation for health professions education: a systematic review and meta-analysis. JAMA. 2011; 306 (9): 978-988.

5. Cummings SR, Browner WS, Hulley SB. Conceiving the research question. In: Hulley SB, Cummings SR, Browner WS, et al., editors. Designing clinical research. 3rd ed. Philadelphia: Lippincott Williams \& Wilkins; 2007. pp. 17-26.

6. Rosenthal R, Schafer J, Briel M, Bucher H, Oertli D, Dell-Kuster $\mathrm{S}$. How to write a surgical clinical research protocol: literature review and practical guide. Am J Surg. 2014; 207 (2): 299-312.

7. Vallejo M. El diseño de investigación: una breve revisión metodológica. Arch Cardiol Méx. 2002; 72 (1): 08-12

8. Manterola C, Otzen T. Estudios observacionales: los diseños utilizados con mayor frecuencia en investigación clínica. Int J Morphol. 2014; 32: 634645.

9. Arguedas-Arguedas O. Tipos de diseño en estudios de investigación biomédica. Acta Méd Costarric. 2010; 52 (1): 16-18.

10. Süt N. Study designs in medicine. Balkan Med J. 2014; 31 (4): 273-277. doi: 10.5152/balkanmedj.2014.1408.

11. Manterola C, Otzen T. Estudios experimentales 2 parte: estudios cuasi-experimentales. Int J Morphol. 2015; 33 (1): 382-387. https://dx.doi.org/10.4067/ S0717-95022015000100060.

12. Manterola C, Otzen T. Los sesgos en investigación clínica. Int J Morphol. 2015; 33 (3): 1156-1164. http:// dx.doi.org/10.4067/S0717-95022015000300056.
13. Eriksson P, Altermann W, Catuneanu O. Some general advice for writing a scientific paper. J African Earth Sci. 2005; 41 (4): 285-288.

14. Villagrán TA, Harris DP. Algunas claves para escribir correctamente un artículo científico. Rev Chil Pediatr. 2009; 80 (1): 70-78.

15. Manterola DC, Pineda NV, Vial GM. Consideraciones y algunas recomendaciones para escribir un artículo científico en una revista biomédica. Rev Chil Cirugía. 2007; 59 (1): 66-74.

16. Ferriols R, Ferriols F. Escribir y publicar un artículo científico original. Ediciones, S.A. Barcelona: Mayo, 2005.

17. Dixon N. Writing for publication: a guide for new authors. Int J Qual Heal Care. 2001; 13 (5): 417-421.

18. Cetin S, Hackam D. An approach to the writing of a scientific manuscript. J Surg Res. 2005; 128 (2): 165167.

19. Kallet R. How to write the methods section of a research paper. Respir Care. 2014; 49 (11): 12291232.

Conflicto de intereses: los autores no refieren conflicto de intereses.

\author{
Correspondencia: \\ Marcia Corvetto A \\ Marcoleta 377, 4을 piso, \\ Santiago, Chile, 6510260. \\ Tel: +5622639-8766/ + 5622354-3270 \\ Fax: +5622632-7620 \\ E-mail: marciacorvetto@gmail.com
}

